\title{
Comparison of 1 versus 3 Days Post-operative Catheterization after Anterior Colporrhaphy
}

\author{
Ahmadi Shahnaz ${ }^{1,2,}$ Rahmani Elham ${ }^{2 *}$, Ahmadi Kambiz ${ }^{3}$ and Kalani Sara ${ }^{2}$ \\ 'Department of Gynecologic Obstetric, Iran University of Medical Sciences, Tehran, Iran; \\ Ahmadishahnaz2005@yahoo.com, \\ 2Department of Gynecologic Obstetric, Bushehr University of Medical Sciences, Bushehr, Iran; \\ rahmani@bpums.ac.ir \\ ${ }^{3}$ Department of Statistics, University of Birjand, Iran; k_ahmadi_stat@yahoo.com
}

\begin{abstract}
With attention to the fact that after anterior colporrhaphy surgery and stitches on vaginal mucus, inserting urinary catheter is requested, according to scientific references and in order to prevent from urinary retention, it has been said that the catheter should be inserted for 3 days. But inserting the catheter leads to infections of bladder and pain. So, in the present study, we compared the removal of the catheter 24 hours versus 72 hours after the surgery, considering urinary infections, urinary retention, patient's ease and hospitalization. Within two groups of 70 patients, which has been assimilated based on basic factors (devided into two groups accidently with excel table) urinary catheter fixed for 1 and 3 days. After that, patients were compared considering urinary retention, urinary infections and the rate of patient satisfaction. Urinary retention was $28.6 \%$ in the test group and $22.9 \%$ in a controlling group which was not statistically meaningful ( $\mathrm{P}=0.78)$. Urinary infections were $22.9 \%$ in the test group and $34.3 \%$ in the controlling group $(\mathrm{P}=0.42)$ and the rate of patient's satisfactions was $57.1 \%$ in the test group and $40 \%$ in controlling group $(\mathrm{P}=0.23)$. The hospitalization period in the test group and in the control group was $2.91 \pm 0.61$ and $3.94 \pm 0.59$ days respectively with a significant difference $(\mathrm{P}=0.001)$. The results showed that short time catheterization is more satisfactory in comparison long catheterizations.
\end{abstract}

Keywords: Anterior Colporrhaphy, Surgery, Urinary Infections, Urinary Retentions

\section{Introduction}

Pelvic organ prolapse is a common age-related disease ${ }^{1,}$ ${ }^{2}$. Its rate of occurrence increases with age ${ }^{2,3}$. A survey conducted, showed that about $12 \%$ of women are possibly exposed to surgery during their life in order to recover from prolapse $e^{2,3}$. The anterior wall of the pelvis is the most common site for prolapse, it accounts for eighty percent of cases ${ }^{4}$.

Prolapse has various causes, and is associated with weakness of the connective tissue and pelvic muscles as well as neural damage ${ }^{4}$. Patients may have no symptoms or they may experience some special symptoms such as low urination, pelvic pain, and some problems related to kidney acceptance, excretion incontinence, backache and dyspareunia ${ }^{4}$.

Non-surgical therapeutic approaches of prolapse include reinforcement of the pelvic muscles and surgical therapy, which consists of a multi-dimensional approach, such that each patient has her private treatment ${ }^{4}$. Moreover, regarding the previous treatments received by the patients, and the level of their activity and health conditions, this therapy differs with each patient.

It is predicted that the necessity of prolapse treatment will increase over the next thirty years to about $45 \%$ due to increase in the population of women above 50 years and excessive parturition ${ }^{2,4}$. The most dangerous factor for prolapse is vaginal delivery. After comparing parameters such as age, race, and body mass index, it is found that women with at least a vaginal delivery are affected by prolapse twice more than women without parturition. Other risk factors for prolapse include hysterectomy, overweight, previous record of prolapse surgery, and race $^{4}$.

*Author for correspondence 
Prolapse is often recognized by urinary disorder symptoms such as excretion incontinence, urinary block symptoms, urinary frequency and urgency; on the other hand, other symptoms like urinary retention, kidney function disorder, pain or anuria may appear. ${ }^{4}$. Other symptoms for prolapse consist of pelvic pain, some problems with kidney acceptance (such as irregularity, dribbling, tenesmus, and excretion incontinence), backache, pelvic pain, and dyspareunia ${ }^{1,4}$. Treatment choice usually depends on the severity of the symptoms and the amount of prolapse along with the general health of the patient and the level of their activity.

To evaluate the anterior and posterior compartment of prolapse, the speculum can be used. During the evaluation of each compartment, each patient is asked to do maneuver Valsalva, in order to determine the whole extent of prolapse. If the result of the Valsalva method is not in harmony with the description of the patient or with her symptoms, doing an examination in standing form while forcing an empty bladder is a useful method. The stages of prolapse are shown in Table 1.

Table 1. The stages of prolapse

\begin{tabular}{ll}
\hline Stage & The grade of prolapse \\
\hline 0 & There is no observed prolapse \\
1 & The lowest part of prolapse is more than $1 \mathrm{~cm}$ \\
& above the hymen level \\
2 & The lowest part of prolapse is less than $1 \mathrm{~cm}$ \\
& proximal or distal to hymen level \\
3 & $\begin{array}{l}\text { The lowest part of prolapse is less than } 1 \mathrm{~cm} \\
\text { under the hymen level but it is not more than } \\
\end{array}$ \\
2 & Full or almost full protrusion of the vagina \\
\hline
\end{tabular}

Studies have shown that patients suffering from prolapse show the absolute spectrum of urinary symptoms, and sometimes some patients may not show any clear symptoms, the most important point is that the objective information about the function of the bladder and urethra must be taken ${ }^{4}$. The basic aim of prolapse surgery is the improvement of those symptoms created by prolapse, and in most cases, restitution of vaginal normalcy, as well as the sexual function may be restored and retained without any complications ${ }^{5}$. The surgical treatment of the vagina is related to the laparotomy or laparoscopy or a combination of these two ${ }^{4}$.

Complications of anterior colporrhaphy: After anterior colporrhaphy surgery, some symptoms, including swellings, hardening, and pains in muscles of sexual and urinary parts may be created ${ }^{5-7}$. After that, an excessive stretch may occur, resulting in destruction of bladder muscle tone (Atony), consequently urinary retention occurs ${ }^{6}$. Urinary retention is the most common complication and its occurrence differs in various studies ${ }^{4,8}$. However, it has been reported between 5 to $70 \%$ in other prevalence studies ${ }^{9}$. The existence of completely different studies about the rate of urinary retention prevalence after surgery is because of different etiologies in this issue and lack of the same criteria for its evaluation. The most exact method for evaluating the amount of urine in the bladder is ultrasound; this can be a proper guidance for assessing the urinary retention after surgery ${ }^{9}$.

Urinary retention after surgery is estimated by measuring if the quantity of urine retained is more than or equal to $200 \mathrm{ml}$ by ultrasound $8 \mathrm{~h}$ after removing catheters $^{10,11}$, or the remaining urine was estimated at 150 CC for patients who cannot empty their bladder 6 hours after removing the catheters ${ }^{12}$. Other complications related to surgery of anterior colporrhaphy are cathetersdependent urinary infections ${ }^{13,14}$ that must be taken into consideration in estimating the time needed for keeping the catheters, because it is estimated as the cause of more than $30 \%$ of hospital infections ${ }^{15}$. Therefore, the urinary catheters are improvised for several days in order to prevent complications after surgery ${ }^{16}$.

Along with reaching the lowest rate of complications, a study has been designed in which the standard and long-term catheterization for three days is compared with a one-day or short-term catheterization after the surgery of anterior colporrhaphy from women hospitalized in Martyrs Hospital in the Persian Gulf in 2013-2015.

\section{Materials and Methods}

In a prospective randomized clinical trial, which was performed on 70 women candidates for anterior colporrhaphy surgery who were admitted into Martyrs Hospital in Persian Gulf in Bushehr, a written informed consent was obtained from all patients after full explanation of the procedure. The study was also approved by the Institutional Ethical Review Board. Those patients who complained about vaginal prolapse were examined with speculum in lithotomy condition using maneuver Valsalva. After confirming prolapse 
based on the inclusion and exclusion criteria, the patients were studied. The inclusion criteria included prolapse of vaginal anterior with grades 2 and 3, age between 25 to 49 years old, and body mass index of $19-24 \mathrm{~kg} / \mathrm{m}^{2}$.

The exclusion criteria consisted of vaginal anterior prolapse grade 1 and 4, Diabetes, connective tissue diseases, different kinds of true urinary incontinence, having a history of hysterectomy. Urine analysis and culture examination was done prior to surgery in all patients. The presence of positive urinary culture or more than 100000 colony counts in each milliliter of urine or more than 10 pieces of leukocyte in each microscopy field was considered as a urinary infection. Patients with urinary infection were omitted from the study. Candidate patients for surgery were given an enema at night before the surgery and all patients were treated with prophylaxis thirty minutes before surgery. The surgery was done by one surgeon and by using the delay absorb thread No. zero and with general anesthesia. After the surgery, the antibiotic was not regularly given except for patients who had abnormal urinary symptoms and unusual urinary analysis in urinary sample $48 \mathrm{~h}$ after the surgery.

Patients were randomized into two groups using computer-generated randomized schedules. In case group (35 patients), urine catheter removed $24 \mathrm{~h}$ after surgery, and in control group (35 patients), urine catheter removed $72 \mathrm{~h}$ after surgery. All the patients whose remaining urine was more than 200 CC eight hours after removal of the catheter or those patients who in spite of urinary necessity were unable to urinate and there was more than 150 CC urine in their bladder from ultrasound result, the urinary catheter were again fixed for $24 \mathrm{~h}$. Patients that lack urinary retention symptoms after removing the catheter were released. Some parameters such as the patients' staying time, the level of their satisfaction, and the rate of urinary infections were examined after surgery.

\section{Statistical Analysis}

Statistical analysis was done by SPSS software edition 18. In order to study the normality of dependent variables (such as age, the hospitalized time), the Kolmogorov-Smirnov test was applied. In cases with an unusual distribution of dependent variables, independent non-parametric T-test Mann-Whitney $U$ was used. The results were explained in the form of mean \pm SD. The means between the two groups were compared by independent T-test. In addition, Fisher exact test was applied for comparing qualitative variables between two groups.

\section{Results}

This study compared the effect of one-day catheterization with three-day catheterization from the rate of urinary retention and urinary infection point of view. Along with reaching this aim, the two groups must be parallelized based on their age, body mass index, and the level of anterior prolapse of the vagina, so that the research just studies the mentioned factors. In this study, the agerelated mean in one-day and three-day catheterization groups were $39.4 \pm 3.2$ years old and $38.8 \pm 2.8$ years old, respectively, and statistically there was no significant difference between them $(\mathrm{P}=0.407)$.

$34.3 \%$ of patients in one-day prolapse group with grade 2 and $31.4 \%$ of them in three-day prolapse group with grade 2 had vaginal anterior phlegm with no significant difference $(\mathrm{P}=0.999)$. The two mentioned groups showed no significant difference with respect to the body mass index $(\mathrm{P}=0.099)$ (Table 2 and Table 3$)$.

Table 2. Demographic of patients in two group

\begin{tabular}{lccc}
\hline Variable & $\begin{array}{c}\text { Test group } \\
(\text { Mean } \pm \text { SD })\end{array}$ & $\begin{array}{c}\text { Control group } \\
(\text { Mean } \pm \text { SD })\end{array}$ & P-value \\
\hline Age & $39.4 \pm 3.2$ & $38.8 \pm 2.8$ & 0.407 \\
BMI & $20 \pm 2.1$ & $20 \pm 2.4$ & $>0.999$ \\
\hline
\end{tabular}

The $\mathrm{p}$-values are obtained using T-test.

Table 3. The grade of anterior prolapses in two groups

\begin{tabular}{lccc}
\hline Variable & Treatment group & Control group & P-value \\
\hline Prolapse 2 & $12(34.3 \%)$ & $11(31.4 \%)$ & $>0.999$ \\
Prolapse 3 & $23(65.7 \%)$ & $24(68.6 \%)$ & \\
\hline
\end{tabular}

The P-value is obtained using Fisher exact test.

These two groups were compared regarding the frequency of urinary retention from each other after surgery in the case group (with one-day catheterization) and in the control group (with three-day urinary catheterization), the result showed $28.6 \%$ and $22.9 \%$ urinary retention respectively with no significant difference between the two groups $(\mathrm{P}=0.78)$. Although the necessity of repeating the catheterization process was higher in the case group (31.4\% against $25.7 \%$ ) but there 
was no significant difference $(\mathrm{P}=0.79)$. As a result, the percentage of patients suffering from urinary infection that tested positive from culture in the three-day group (34.3\%) was also higher than the one-day group (22.9\%), but showed no significant difference statistically $(\mathrm{P}=0.42)$. Moreover, the patients' level of satisfaction about catheterization and their hospitalization was higher in the test group, but showed no significant difference (57.1\% against $40.0 \%)$.

The hospitalization period in test group and in control group was $2.91 \pm 0.61$ and $3.94 \pm 0.59$ days respectively with a significant difference $(\mathrm{P}=0.001)$ (Table 4$)$.

Table 4. A comparison of consequences in urinary retention, second catheterization, positive urinary culture, and the patients' level of satisfaction from the period of hospitalization between test and control group after anterior colporrhaphy surgery

\begin{tabular}{lcrrr}
\hline Variable & & Test group & $\begin{array}{c}\text { Control } \\
\text { group }\end{array}$ & P-value \\
\hline Urinary retention & Yes & $10(28.6 \%)$ & $8(22.9 \%)$ & 0.78 \\
& No & $25(71.4 \%)$ & $27(77.1 \%)$ & \\
Re-catetrization & Yes & $11(31.4 \%)$ & $9(25.7 \%)$ & 0.79 \\
& No & $24(68.6 \%)$ & $26(74.3 \%)$ & \\
Positive urine & Yes & $8(22.9 \%)$ & $12(34.3 \%)$ & 0.43 \\
culture & No & $27(77.1 \%)$ & $23(65.7 \%)$ & \\
Patient satisfaction & Yes & $20(57.1 \%)$ & $14(40.0 \%)$ & 0.23 \\
& No & $15(42.9 \%)$ & $21(60.0 \%)$ & \\
\hline
\end{tabular}

The P-values are obtained using Fisher exact test.

\section{Discussion}

A study by Hakvoort in 2004 on this subject in which urinary catheters were inserted for four days for a group and removed on the fifth day. In another group, the catheters were removed one day after the surgery. In addition, some factors, such as the amount of urine retained, urinary infection and the period of hospitalization were compared between two groups. The level of urinary retention in the one-day group is higher than the fourday group, but the level of their urinary infection and the period of their hospitalization were less than the four-day group. It is known that the disadvantages of long-term catheterization are more than its advantages; hence it must be done just when it is really needed ${ }^{17}$. Thakour et al. conducted a study in the women's room of the educational hospital TU in Maharajguni, on 100 patients and the oneday and three-day catheterization was compared. The results obtained from this study showed that the level of urinary infection in the short-term catheterization group was lower than the other; the rate of urinary retention in this group was also found to be higher than the other. However, urinary retention has not been reported in the long-term catheterization group ${ }^{18}$.

In another study conducted by Hakvoortin, the catheterization was done after the surgery for all patients, and their catheters were removed after one day. Those patients whose urine content was more than $150 \mathrm{ml}$ were divided into two groups, one of them used catheters when they needed to urinate, while another one improvised the catheters for three days. In the first group, the amount of urinary infection was $12 \%$ but its amount in the second group was $33 \%$, so the first group needed a very short time for catheterization. The level of patients' satisfaction was the same in the two groups ${ }^{19}$.

Another research was performed by Wanderstin in 2010 using two groups; they improvised the catheters for one day and three days, respectively. The results showed that the period of catheterization and hospitalization in the first group was significantly decreased, but in the second group, the level of urinary retention was less than the other (3.79\% versus 9.90\%). However, the level of urinary infection was not significantly different in two groups $^{20}$.

Also, in this case, a research was conducted by Weemhoof, in which the removal of urinary catheters was compared between two to five days, after anterior colporrhaphy surgery. It was concluded that removing the catheters in the second day required further improvising compared to the fifth day, but the level of urinary infection and the period of hospitalization was less in this group ${ }^{21}$.

In other research, Adly concluded that in the group in which the urinary catheters were removed earlier, they needed further catheterization three to four times more than the other group; whereas in the second group whose catheters were removed after five days, the probability of urinary infection was 14 times more than the other. It was concluded that removing the catheters in the first $24 \mathrm{~h}$ is more appropriate ${ }^{10}$.

In recent studies performed by Joshi and Aggarwal, on seventy patients, the short-term and long-term catheterizations were compared regarding the level of urinary infection and urinary retention. Thus, they concluded that the short-term catheterization does not increase the level of urinary infection, and the patients in this group felt less pain and illness compared to the others. However, the rate of further catheterization was 
higher in this group than the other, but the difference was not statistically clear ${ }^{22}$.

According to different studies, the urinary catheters were removed on the first, second, fourth, and fifth days, as well as $3 \mathrm{~h}$ after surgery or even immediately after prolapse surgery ${ }^{23}$. Although based on these studies, the period of keeping the catheters is challenging, in a research published by Cochrane, it was determined that when the catheters were removed earlier, the level of urinary infection was lower in seven out of eleven studies ${ }^{24-28}$.

In our research, the two groups recorded no significant difference from the frequency of urinary retention point of view from each other $(\mathrm{P}=0.78)$. Despite the necessity for repetition of the catheterization process, it was more in the one-day group than the others (31.8\% against $25 \%)$, there was no significant difference in this case $(\mathrm{P}=0.79)$. Whereas in other studies, the level of urinary retention in the one-day group was more than the three-day group. The reason for the lack of difference might be because all the patients had cystocele grade 2, and the intense cystocele had been omitted from the study.

In the present research, the percentage of patients suffering from urinary infection and having a positive culture result in the three-day group $(38.8 \%)$ was also higher than the one-day group (22.7\%), but this difference was not statistically significant $(\mathrm{P}=0.42)$ this may be due to the small size of the studied sample. In this study, the average hospitalization period in the oneday catheterization group and the three-day group was $3.91 \pm 0.61$ and $4.95 \pm 0.59$ days respectively and there was a significant difference between them $(\mathrm{P}=0.0001)$. Consequently, the patients' level of satisfaction in one-day catheterization group was more than the three-day group (57.1\% against $40 \%$ in the three-day group), but it was not statistically significant $(\mathrm{P}=0.001)$.

\section{Conclusion}

In this study, short-term or one-day catheterization increased the level of patients' satisfaction compared to the group whose patients kept the catheters for several days $(\mathrm{P}=0.001)$. However, in other aspects such as decreasing the level of urinary infection, there was no significant difference between these two groups $(\mathrm{P}=0.42)$. Also, there was no significant difference between them from evaluating the urinary retention $(\mathrm{P}=0.78)$. Therefore, it seems that although the short-term catheterization showed no remarkable results from the decline in the level of urinary infection, it increased the level of patients' satisfaction.

\section{Acknowledgements}

The authors are grateful to the Bushehr University of Medical Sciences, the Vice chancellery of Research and Technology Affairs, that approved the proposal and this data was collected from Miss Kalani's thesis. IRCT201506134339N11.

\section{References}

1. Toz M, Özcan A, Apaydın N, Uyar I, Kocakaya B, Okay G. Outcomes of vaginal hysterectomy and constricting colporrhaphy with concurrent levatormyorrhaphy and high perineorrhaphy in women older than 75 years of age. Dove Press J. 2015; 10:1009-15.

2. Nüssler E, Kesmodel US, Löfgren M. Operation for primary cystocele with anterior colporrhaphy or non-absorbable mesh: patient-reported outcomes. Int Urogynecol J. 2015; 26:359-66.

3. Swenson CW, Simmen AM, Berger MB, Morgan DM, Delancey IO. The long and short of it: anterior vaginal wall length before and after anterior repair. Int Urogynecol J. 2015; 26:1035-9.

4. Gleason LG, Richter HE, Varner RE. Pelvic organ prolapse. In: Berek JS, Novak E, editors. Berek \& Novak's gynecology. 15th ed. Philadelphia: Lippincott Williams \& Wilkins; 2012. p. 176-208.

5. Rock JA, Jones HW, III. Telindes operative gynecology. 11th ed. Philedaphia: Lippincott Williams \& Wilkins; 2015. p. 928-40.

6. Brunicardi FC, Anderson DK. Schwartz' principles of surgery. 10th ed. New York: McGrawhill; 2014. 154.

7. Pant PR. An effective short duration postoperative catheterization after vaginal hysterectomy and pelvic floor repair. J Institute Med. 2006; 28(1):33-5.

8. Chai J, Pun TC. A prospective randomized trial to compare immediate and 24-hour delayed catheter removal following total abdominal hysterectomy. Acta Obstet Gynecol Scand. 2011; 90(5):478-82.

9. Baldini G, Bagry H, Aprikian A, Caril F. Postoperative urinary retention. Anesthesiology. 2009; 110:1139-57.

10. Adly N, Fattah A, Budi I. Urinary catheterization in gynecological surgery: When should it be removed? Med J Indones. 2013; 22:183-8.

11. Kamilya G, Seal SL, Mukherji J, Bhattacharyya SK, Hazra A. A randomized controlled trial comparing short versus long-term catheterization after uncomplicated vaginal prolapse surgery. J Obstet Gynaecol Res. 2010; 36(1):154-8. 
12. Chai J, Pun TC. A prospective randomized trial to compare immediate and 24-hour delayed catheter removal following total abdominal hysterectomy. Acta Obstet Gynecol Scand. 2011; 90(5):478-82.

13. Doppa GJ, Gowder RO, Munaganur N. A rospective study comparing short term catheterization versus non catheterization of bladder following vaginalhysterectomy. JEMDS. 2014; 3:1308-14.

14. Baldini G, Bagry H, Aprikian A, Caril F, Phil M. Postoperative urinary retention. Anesthesiology. 2009; 110:1139-57.

15. Ercole FF, Macieira TGR, Wenceslau L, Martins AR, Campos C, Chianca T. Evidences on the practice of intermittent/ indwelling urinary catheterization. Rev Latino-Am Enfermagem. 2013; 21(1):459-68.

16. Movahed F, Rahmani L. Compairing short term and long term catheterization after anterior colporrhaphy. Journal of Hamedan University of Medical Science. 2010; 56:41-4.

17. Hakvoort RA, Elberink R, Vollebregt A, Ploeg VD, Emanuel $\mathrm{MH}$. How long should urinary bladder catheterization be continued after prolapse surgery? A randomized controlled trial comparing short term versus long term catheterisation after vaginal prolapse surgery. Br J Obstet Gynecol. 2004; 111(8):828-30.

18. Thakur N, Gurung G, Rana A. A randomized controlled trial comparing short term versus long term catheterization after vaginal prolapse surgery. N J Obstet Gynaecol. 2007; 2(1):29-34.

19. Hakvoort R, Thijs SD, Bouwmeester FW, Brookman AM, Ruhe IM, Vernooij MM, Burger MP, Emanuel MH, Roovers JP. Comparing clean intermittent catheterisation and transurethral indwelling catheterisation for incomplete voiding after vaginal prolapse surgery: a multicenter randomised trial. BJOG. 2011; 118:1055-60.

20. Vandersteen A, Detollenaere R, den Boon J, van Eijndhoven $\mathrm{H}$. One-day versus 3-day suprapubic catheterization after vaginal prolapse surgery: a prospective randomized trial. Int Urogynecol J. 2011;22:563-567.

21. Weemhoff M, Wassen MMLH, Korsten L, Serroyen J, Paul HTNK, Frans JMER. Postoperative catheterization after anterior colporrhaphy: 2 versus 5 days. A multicentre randomized controlled trial. Int Urogynecol J. 2011; 22:477-83.

22. Joshi B, Aggarwal N, Chopra S, Taneja N. A prospective randomized controlled comparison of immediate versus late removal of urinary catheter after abdominal hysterectomy. J Mid-life Health. 2014; 5:68-71.

23. Glavind K, Morup L, Madsen H, Glavind J. A prospective, randomized, controlled trial comparing 3 hour and 24 hour postoperative removal of bladder catheter and vaginal pack following vaginal prolapse surgery. Acta Obstet Gynecol Scand. 2007; 86:1122-5.

24. Phipps S, Lim YM, McClinton S, Barry C, Rane A, N’Dow JMO. Short term urinary catheter policis following urogenital surgery in adults. Cochrane Database of Syst Rev. 2006; 2:CD004374.

25. Eftekhar M, Rahmani E, Pourmasum S. Evaluation of clinical factors influencing pregnancy rate in frozen embryo transfer. Iran J of Reprod Med. 2014; 12:513-8.

26. Eftekhar M, Firouzabadi RD, Karimi H, Rahmani E. Outcome of cryopreserved-thawed embryo transfer in the $\mathrm{GnRH}$ agonist versus antagonist protocol. Iran J of Reprod Med. 2012; 10 (4): 297-302.

27. Eftekhar M, Khalili MA Rahmani E. The efficacy of recombinant versus urinary HCG in ART outcome. Iran J Reprod Med. 2012; 10:543-8.

28. Tajbakhsh S, et al. Identification of Streptococcus agalactiae by fluorescent in situ hybridization compared to culturing and the determination of prevalence of Streptococcus agalactiae colonization among pregnant women in Bushehr, Iran. BMC Infect Dis. 2013; 13(1): 420. 